\title{
DATOS SOBRE LA VEGETACIÓN DE LOS HUMEDALES DE AMÉRICA DEL SUR. DE LAS SABANAS BOLIVIANAS A LOS LLANOS DEL ORINOCO (VENEZUELA)
}

\author{
Antonio GALÁN DE MERA ${ }^{1 *}$ y Eliana LINARES PEREA ${ }^{2}$ \\ ${ }^{1}$ Departamento de Ciencias Ambientales y Recursos Naturales, Facultad de Farmacia, Universidad \\ San Pablo-CEU, Apartado 67, 28660- Boadilla del Monte, Madrid, España \\ ${ }^{2}$ Universidad Nacional de San Agustín, Sánchez Cerro 219, Manuel Prado, \\ Paucarpata, Arequipa, Perú \\ *Autor para correspondencia: agalmer@ceu.es
}

Recibido el 19 de noviembre de 2007, aceptado para su publicación el 10 de abril de 2008 Publicado "on line" en junio de 2008

RESUMEN. Datos sobre la vegetación de los humedales de América del Sur. De las sabanas bolivianas a los Llanos del Orinoco (Venezuela). En base a los inventarios fitosociológicos levantados en 3 humedales de América del Sur (Llanos de Moxos y Pantanal, Bolivia; Llanos del Orinoco, Venezuela) damos a conocer semejanzas y diferencias en la composición de comunidades vegetales. Como resultado, describimos 4 asociaciones [Hydrocleydo nymphoidis-Nymphoidetum herzogii (comunidades de ninfeidos), Oxycaryo cubensis-Eleocharitetum acutangulae (graminales flotantes), Ipomoeo fistulosae-Sennetum aculeatae (vegetación nitrófila) y Bacopo myriophylloidis-Eleocharitetum minimae (pastizales inundables)] y una alianza (Echinodorion boliviani). Entre los humedales observados, 9 comunidades vegetales son comunes; Ludwigio sedoidis-Eichhornietum diversifoliae (comunidades de ninfeidos), Spilantho uliginosi-Paspaletum orbiculati (pastizales inundables) y Thalietum geniculatae (asociación de grandes helófitos) son características de los Llanos del Orinoco, mientras que Bacopo myriophylloidis-Eleocharitetum minimae, comunidades de Pontederia parviflora (comunidades helofíticas de aguas someras), Hydrocleydo-Nymphoidetum herzogii y RhabdadenioCyperetum gigantei (asociación de grandes helófitos) lo son de los llanos neotropicales situados al S de la Cuenca Amazónica.

Palabras clave. Vegetación, sintaxonomía, Bolivia, Venezuela, humedales.

ABSTRACT. Data about the vegetation of the wetlands of South America. From the Bolivian savannas to the Llanos of the Orinoco (Venezuela). Based on the phytosociological relevés made up in 3 wetlands of South America (Llanos of Moxos and Pantanal, Bolivia; Llanos of the Orinoco, Venezuela), similarities and differences in the composition of plant communities are presented. As a result, 4 associations [Hydrocleydo nymphoidis-Nymphoidetum herzogii (water lily communities), Oxycaryo cubensis-Eleocharitetum acutangulae (floating grasslands), Ipomoeo fistulosae-Sennetum aculeatae (nitrophilous vegetation) and Bacopo myriophylloidis-Eleocharitetum minimae (flooding pastures)] and one alliance (Echinodorion boliviani) are described. In the observed wetlands, 9 plant communities are commons; Ludwigio sedoidis-Eichhornietum diversifoliae (water lily communities), Spilantho uliginosi-Paspaletum orbiculati (flooding pastures) and Thalietum geniculatae (high helophytic vegetation) are characteristics of the Llanos of the Orinoco, while Bacopo myriophylloidisEleocharitetum minimae, Pontederia parviflora plant communities (communities of shallow water), 
Hydrocleydo-Nymphoidetum herzogii and Rhabdadenio-Cyperetum gigantei (high helophytic vegetation) are presents in the Neotropical lands placed to the southern of Amazonian Basin.

Key words. Vegetation, syntaxonomy, Bolivia, Venezuela, wetlands.

\section{INTRODUCCIÓN Y OBJETIVOS}

Los humedales de América del Sur son áreas que alcanzan una gran diversidad, y que por su composición florística y situación geográfica podríamos dividir en 4 grupos:

1- Humedales andinos neotropicales (Cleef, 1981; Rangel \& Aguirre, 1983; Franco et al. 1986; Seibert \& Menhofer, 1991, 1992; Galán de Mera et al. 2003, 2004)[algunas plantas características: Distichia muscoides Nees \& Meyen, Eleocharis macrostachya Britton, Isoetes andicola (Amstutz) L.D. Gómez, I. glacialis Aspl., Juncus ecuadoriensis Balslev, Myriophyllum quitense H.B.K., Oxychloe andina Phil., Potamogeton illinoensis Morong, Puccinellia frigida (Phil.) I.M. Johnst., Ranunculus limoselloides Turcz., Scirpus californicus subsp. tatora (Kunth) T. Koyama, Stuckenia punensis (A. Galán) A. Galán],

2- Humedales andinos patagónicos y de clima templado (Boelcke et al. 1985; San Martín Padovani et al. 1993; Hauenstein et al. 2002; Gandullo \& Faggi, 2005)[algunas plantas características: Caltha sagittata Cav., Carex gayana Desv., C. magellanica Lam., Deyeuxia neglecta Kunth, Egeria densa Planch., Glyceria multiflora Steud., Hippuris vulgaris L., Hordeum lechleri (Steud.) Schenck, Potamogeton berteroanus Phil., Myriophyllum aquaticum Verdc., Nymphaea alba L.],

3- Humedales de la Cuenca Amazónica y de las sabanas inundables del Orinoco, Mato Grosso y Moxos (Haase, 1989, 1990;
Encarnación, 1993; Kalliola et al. 1993; Colonnello, 1995; Galán de Mera, 1995, 2007; Fuentes \& Navarro, 2000)[algunas plantas características: Eichhornia azurea (Sw.) Kunth, Hymenachne amplexicaulis (Rudge) Nees, Limnobium laevigatum (Humb. \& Bonpl. ex Willd.) Heine, Montrichardia arborescens (L.) Schott, Nymphaea amazonum Mart. \& Zucc., Pontederia subovata (Seub.) Lowden, Thalia geniculata L., Utricularia foliosa L., U. gibba L., Victoria amazonica (Poepp.) J.C. Sowerby],

4- Humedales de la región desértica pacífica (Müller \& Gutte, 1985; Linares Perea, 2000)[algunas plantas características: Cyperus laevigatus L., Phragmites australis (Cav.) Trin. ex Steud., Pityrogramma trifoliata (L.) R.M. Tryon, Potamogeton striatus Ruiz \& Pav., Rapanea manglillo (Lam.) Mez, Scirpus americanus Pers., Typha domingensis Pers.].

De estos humedales, los de los Llanos del Orinoco $\left(240.000 \mathrm{Km}^{2}\right)$ y el Pantanal brasileño, boliviano y paraguayo $(280.000$ $\mathrm{Km}^{2}$ ) son los más extensos del mundo (Adámoli, 1999; Convention on Wetlands, 2007). Incluso este último autor indica al Pantanal como el humedal más diverso del mundo. Lo cierto es que tanto el Pantanal (parte brasileña) como los Llanos del Orinoco -y por extensión el Delta del Orinoco- tal vez sean los más estudiados (Sarmiento \& Monasterio, 1969; Prance \& Schaller, 1982; Castroviejo \& López, 1985; Ratter et al. 1988; Pott et al. 1989; Pott \& Pott, 1994, 2000; Colonnello, 1995; Zeilhofer \& Schessl, 1999; Rial, 2000; Galán 
de Mera et al. 2006; Galán de Mera, 2007).

Esta abundancia de referencias nos puede introducir a la comparación entre áreas de América del Sur con similitudes geomorfológicas y vicarianzas florísticas. Así, los aportes de Galán de Mera \& Navarro (1992) -Paraguay- Beck (1984), Haase (1989, 1990), Fuentes \& Navarro (2000), Pouilly et al. (2004) -Bolivia- Deil (2005) síntesis mundial de la vegetación de los humedales efímeros- Galán de Mera et al. (2006), Galán de Mera (2007) -Venezuelay los nuevos datos que aportamos sobre el Pantanal y los Llanos de Moxos bolivianos, han permitido establecer una comparación de la vegetación acuática y palustre de un área de la cuenca del Orinoco y localidades de las cuencas del Beni y del Paraguay.

\section{MATERIAL Y MÉTODOS}

El aumento de datos sobre la flora y la vegetación de distintas áreas de América del Sur (ver por ejemplo, Steyermark et al. 1995, 2005; Rangel et al. 1997; Galán de Mera \& Vicente Orellana, 2006) está llevando a adoptar la metodología de comparar la biodiversidad de regiones con las mismas características fisionómicas (Daly \& Mitchell, 2000; Linares-Palomino et al. 2003; Pennington et al. 2006).

Por esta razón, el método fitosociológico (Braun-Blanquet, 1964; Dierschke, 1994), basado en las características florísticas de las comunidades vegetales, ofrece una buena posibilidad para conocer la biodiversidad de regiones disyuntas sintetizando caracteres ecológicos y flora en las tablas fitosociológicas.

\section{Territorio estudiado}

Hemos elegido tres zonas (fig. 1) que representan a los humedales de las sabanas inundables del Orinoco (Estación Biológica

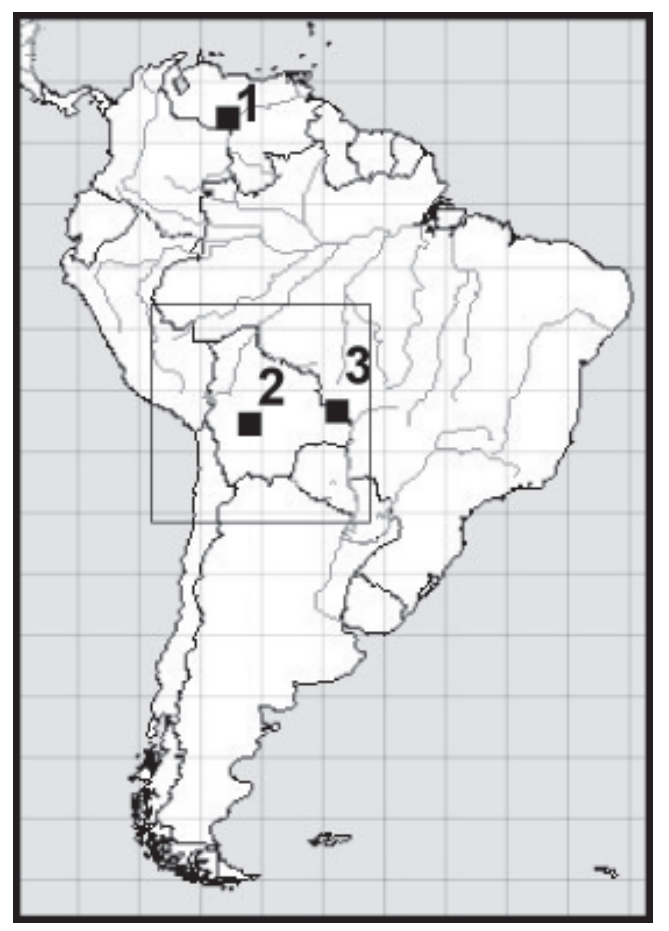

Figura 1. Los territorios estudiados: 1- Estación Biológica El Frío, 2- Estancia Los Reyes, 3Reserva Biológica de El Pantanal. El cuadrado es el área que comprende el mapa de la figura 2. The studied territories: 1- El Frio Biological Station, 2- Los Reyes cattle ranch, 3-El Pantanal Biological Reserve. The square is the included area in the map of the figure 2. (base cartográfica tomada de Missouri Botanical Garden).

El Frío), Moxos (estancia Los Reyes) y Pantanal (Reserva Biológica El Pantanal):

La Estación Biológica El Frío en Venezuela $\left(7^{\circ} 50^{\prime} \mathrm{N}-69^{\circ} \mathrm{O}, 70-80 \mathrm{~m}\right)$ está situada al O de San Fernando de Apure, en la gran llanura inundable arcillosa y limosa cuaternaria de los Llanos del Orinoco, perteneciente a la provincia biogeográfica de los Llanos (infratropical subhúmedo).

La estancia Los Reyes en los Llanos de Moxos en Bolivia (14 $56^{\prime} \mathrm{S}-66^{\circ} 47^{\prime} \mathrm{O}, 160$ $170 \mathrm{~m}$ ) está situada al SO de San Borja, en una llanura arcillosa cuaternaria de la cuenca 
del río Beni (tributario del Amazonas), que podemos incluir en la provincia biogeográfica del Cerrado, sector de los Llanos de Moxos (termotropical subhúmedo).

La Reserva Biológica El Pantanal en Bolivia (16²66'S-5851'O, $97 \mathrm{~m}$ ) está situada al S de San Matías, en las arenas y arcillas del Gran Curichi fronterizo con Brasil, tributario del río Paraguay. Es una llanura de mayor extensión en Brasil- que igualmente incluimos en la provincia del Cerrado, sector del Pantanal (infratropical subhúmedo).

\section{Bioclimatología}

En la tabla 1 y en la figura 2 se representan las estaciones meteorológicas más próximas a los territorios estudiados y su diagnosis bioclimática siguiendo el modelo propuesto por Rivas-Martínez et $a l$. (1999). Los datos meteorológicos proceden de Müller (1982) y Rivas-Martínez (2005).

Excepto Corumbá (1) y Puerto Suárez (3), que tienen carácter termotropical seco, debido a que son más orientales, todas presentan ombroclima subhúmedo. San Borja (4) sigue siendo termotropical debido a la influencia de los vientos del S, que producen una caída de las temperaturas durante la estación seca (junio-septiembre).

Por tanto, tanto el territorio estudiado en Venezuela [cercano a San Fernando de Apure (5)] como en el Pantanal boliviano [cercano a Cuiabá (2)] quedan dentro del infratropical subhúmedo; la estancia Los Reyes [cercana a San Borja (4)] corresponde a termotropical subhúmedo.

\begin{tabular}{|c|c|c|c|c|c|c|c|}
\hline Estación meteorológica & $\mathrm{T}$ & M & $\mathrm{m}$ & $\mathrm{P}$ & It & Io & Descripción bioclimática \\
\hline $\begin{array}{l}\text { 1-Corumbá (Brasil), } 138 \mathrm{~m} \\
\left(19^{\circ} 00^{\prime} \mathrm{S}, 57^{\circ} 39^{\prime} \mathrm{O}\right)\end{array}$ & 25,1 & 28,0 & 16,2 & 1063,0 & 693 & 3,5 & Termotropical seco \\
\hline 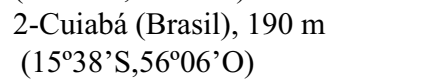 & 27,0 & 31,1 & 17,2 & 1419,7 & 753 & 4,4 & Infratropical subhúmedo \\
\hline $\begin{array}{l}\text { 3-Puerto Suárez (Bolivia), } 152 \text { m } \\
\left(18^{\circ} 59^{\prime} \mathrm{S}, 57^{\circ} 46^{\prime} \mathrm{O}\right)\end{array}$ & 25,6 & - & - & 1047,0 & 675 & 3,4 & Termotropical seco \\
\hline $\begin{array}{l}\text { 4-San Borja (Bolivia), } 193 \mathrm{~m} \\
\left(15^{\circ} 52^{\prime} \mathrm{S}, 66^{\circ} 52^{\prime} \mathrm{O}\right)\end{array}$ & 25,0 & 28,0 & 16,2 & 2039,1 & 692 & 6,7 & Termotropical subhúmedo \\
\hline $\begin{array}{l}\text { 5-San Fernando de Apure } \\
\text { (Venezuela), } 73 \mathrm{~m} \\
\left(07^{\circ} 53^{\prime} \mathrm{N}, 67^{\circ} 26^{\prime} \mathrm{O}\right)\end{array}$ & 27,0 & 25,5 & 25,5 & 1491,0 & 781 & 4,6 & Infratropical subhúmedo \\
\hline
\end{tabular}

Tabla 1. Variables climáticas, índices bioclimáticos y descripción bioclimática de estaciones meteorológicas próximas a los territorios estudiados, de acuerdo con la metodología de Rivas-Martínez (1999)[T: temperatura media anual $\left({ }^{\circ} \mathrm{C}\right), \mathrm{M}$ : temperatura media de las máximas del mes más frío $\left({ }^{\circ} \mathrm{C}\right)$, $\mathrm{m}$ : temperatura media de las mínimas del mes más frío $\left({ }^{\circ} \mathrm{C}\right), \mathrm{P}$ : precipitación media anual $(\mathrm{mm})$, It: índice de termicidad -It $=(\mathrm{T}+\mathrm{M}+\mathrm{m}) 10$-, Io: índice ombrotérmico -Io = P/12T-; pisos bioclimáticos: It = 731-890-infratropical, 491-730-termotropical; intervalos ombrotérmicos: Io = 2,1-3,0-seco, 3,15,5-subhúmedo]. Climatic variables, bioclimatic indices and bioclimatic description of meterorological stations of the surroundings of the studied territories, according to the RivasMartinez's methodology (1999) [T: mean annual temperature $\left({ }^{\circ} \mathrm{C}\right)$, M: mean higuest temperatures of the coldest month $\left({ }^{\circ} \mathrm{C}\right), \mathrm{m}$ : mean lowest temperatures of the coldest month $\left({ }^{\circ} \mathrm{C}\right), \mathrm{P}$ : mean annual precipitation $(\mathrm{mm})$, It: thermicity index $-I t=(T+M+m) 10-$, Io: ombrothermic index $-I o=P / T-$ : bioclimatic belts: $I t=731$-890-infratropical, 491-730-thermotropical; ombrothermic intervals: Io = 2.1-3.0-dry, 3.1-5.5-subhumid]. 


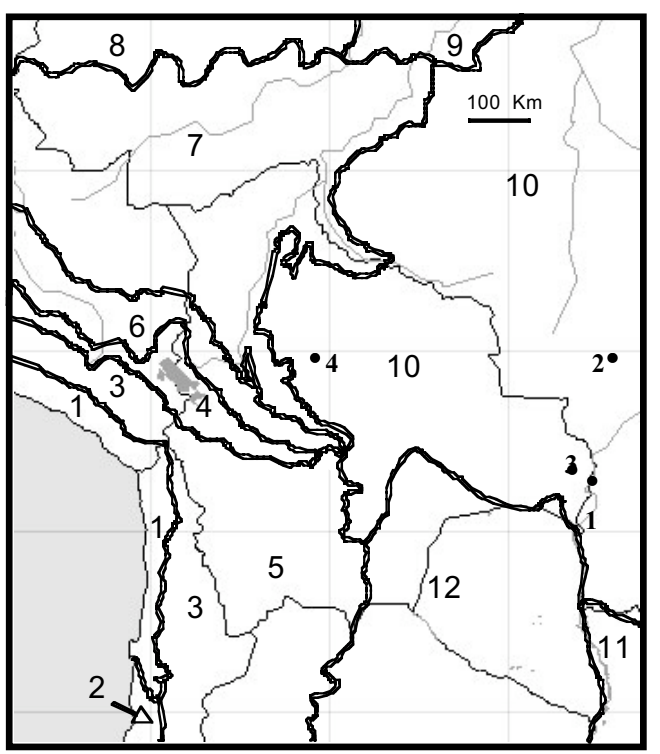

Figura 2. Provincias fitogeográficas del centro de América del Sur. Phytogeographyical provinces of the central South America. 1- Provincia Limeño-Ariqueña, 2- Provincia del Desierto Mesochileno, 3- Provincia Oruro-Arequipeña, 4Provincia Ancashino-Paceña, 5- Provincia Urubambense, 6- Provincia de Madre de Dios, 7- Provincia Loretana, 8- Provincia Madeira y Tapajoz, 9- Provincia del Cerrado, 10- Provincia Boliviano-Tucumana, 11- Provincia del Chaco Boreal, 12- Provincia Paranense. Los puntos negros son las estaciones meteorológicas de la tabla 1. The black points are the meteorological stations of the table 1. (base cartográfica tomada de Missouri Botanical Garden).

\section{Vegetación}

Son predominantes los bosques semidecíduos. En el territorio de los Llanos del Orinoco son frecuentes algunas especies como Bromelia chrysantha Jacq., Ceiba pentandra (L.) Gaertn., Couroupita guianensis Aubl., Ficus guianensis Desv. ex Ham., Platymiscium pinnatum (Jacq.) Dugand, Pterocarpus rohrii Vahl, Samanea saman (Jacq.) Merr. o Spondias mombin L. -Bromelio chrysanthae-Platymiscietum pinnati (Galán de Mera et al., 2006). Sin embargo, en las localidades bolivianas son frecuentes los bosques con una fisionomía similar, con especies de Tabebuia y una elevada cantidad de especies comunes como Cissus spinosa Cambess., Combretum lanceolatum Pohl ex Eichler, Copernicia alba Morong ex Morong \& Britton, Cordia alliodora (Ruiz \& Pav.) O Ken, C. glabrata (Mart.) DC., Curatella americana L., Desmoncus cuyabaensis Barb. Rodr., Licania parviflora Benth., Machaerium hirtum (Vell.) Stellfeld, Tabebuia aurea (Silva Manso) Benth. \& Hook. f. ex S. Moore, T. heptaphylla (Vell.) Toledo y $T$. impetiginosa (Mart. ex DC.) Standl.

\section{Fitogeografía}

En la figura 2 damos a conocer las provincias fitogeográficas del centro de América del Sur, donde se encuentra enmarcado este trabajo, y cuya jerarquía es las siguiente:

\section{Reino Neotropical}

Subreino Caribeo-Amazónico Región Andina

Subregión del Desierto Pacífico

1- Provincia Limeño-Ariqueña

2- Provincia del Desierto Mesochileno

Subregión Paramuno-Puneña

3- Provincia Oruro-Arequipeña

4- Provincia Ancashino-Paceña

5- Provincia Boliviano-Tucumana

Subregión de la Ceja de Montaña

6- Provincia Urubambense

Región Amazónica

7- Provincia de Madre de Dios

8- Provincia Loretana

9- Provincia Madeira y Tapajoz

Región Brasileño-Paranense

10- Provincia del Cerrado

11- Provincia Paranense

Región Chaqueña

12-Provincia del Chaco Boreal

Tomando como referencia los trabajos de 
Tabla 2

\begin{tabular}{|c|c|c|c|c|c|c|}
\hline \multicolumn{7}{|c|}{$\begin{array}{c}\text { Hydrocleydo nymphoidis-Nymphoidetum herzogii } \\
\text { Galán de Mera \& Linares Perea ass. nova } \\
\text { (Cabombo-Nymphaeetea, Nymphaeetalia amplae, Nelumbo-Nymphaeion) }\end{array}$} \\
\hline Inventario $\mathrm{n}^{\mathrm{o}}$ & 1 & 2 & 3 & 4 & 5 & 6 \\
\hline Área $\mathrm{m}^{2}$ & 100 & 25 & 100 & 100 & 100 & 100 \\
\hline Profundidad (cm) & 15 & 10 & 20 & 30 & 10 & 20 \\
\hline \multicolumn{7}{|l|}{ Características de asociación } \\
\hline Nymphoides herzogii & 2 & 1 & 1 & 1 & & 1 \\
\hline Hydrocleys nymphoides & + & 3 & . & . & 2 & . \\
\hline Marsilea crotophora & 4 & 3 & . & . & . & . \\
\hline Utricularia poconensis & . & . & . & . & . & 2 \\
\hline \multicolumn{7}{|c|}{ Características de unidades superiores } \\
\hline Salvinia auriculata & 1 & . & . & 1 & 4 & 3 \\
\hline Utricularia foliosa & . & . & 2 & 1 & 1 & \\
\hline Utricularia gibba & . & 1 & + & . & . & 1 \\
\hline Sagittaria guyanensis & . & . & 2 & . & . & 1 \\
\hline Nymphaea amazonum & . & . & 3 & . & . & 2 \\
\hline Azolla filiculoides & . & + & . & . & . & . \\
\hline Ludwigia helminthorrhiza & . & 2 & . & & . & . \\
\hline Ludwigia sedoides & . & . & . & 1 & . & . \\
\hline \multicolumn{7}{|l|}{ Compañeras } \\
\hline Eichhornia azurea & . & . & 1 & + & 1 & + \\
\hline Eleocharis interstincta & . & . & 1 & 1 & . & 1 \\
\hline Pontederia subovata & 1 & 1 & . & . & . & . \\
\hline Pontederia parviflora & . & . & 1 & 1 & . & . \\
\hline Aeschynomene scabra & + & . & . & . & . & . \\
\hline Hymenachne donacifolia & . & 1 & . & . & . & . \\
\hline Hydrolea spinosa & . & . & + & . & . & 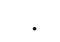 \\
\hline Pontederia cordata var. lancifolia & . & . & . & . & . & 1 \\
\hline
\end{tabular}

Localidades: 1 y 2- Bolivia, Beni, San Borja, Estancia Los Reyes (19L7399); 3 y 4- Bolivia, Santa Cruz, San Matías, Reserva Biológica El Pantanal (21K4057); 5 y 6- Bolivia, Santa Cruz, San Matías, Reserva Biológica El Pantanal (21K4356).

Galán de Mera et al. (2002, 2006) y Navarro \& Maldonado (2005), el esquema biogeográfico de los territorios estudiados queda de la siguiente forma:

\section{Reino Neotropical}

Subreino Caribeo-Amazónico

[Venezuela, Estación Biológica El Frío]

Región Caribeño-Mesoamericana

Provincia de Los Llanos

[Bolivia, estancia Los Reyes y Reserva

\section{Biológica El Pantanal] \\ Región Brasileño-Paranense Provincia del Cerrado}

Como indica Adámoli (1999) la provincia del Cerrado se caracteriza por la presencia de elementos chaqueños (p.ej. Tabebuia sp. pl., Copernicia alba) y, como sugieren Navarro \& Maldonado (2005) los llanos de Moxos (Beni) y del Pantanal podrían incluirse en la provincia del Cerrado. 
Desde nuestro punto de vista, la provincia del Cerrado se extiende tanto a los llanos del Beni como al Pantanal pues se presentan numerosas especies comunes, que ya indicábamos anteriormente.

\section{Nomenclatura}

La nomenclatura y autorías de los táxones tratados están de acuerdo con Galán de Mera (2007) para los Llanos del Orinoco. Las especies bolivianas están basadas en Pott et al. (1989), Killeen et al. (1993), Pott \& Pott (2000) y Pennington et al. (2004). En general, se ha consultado la base de datos W3-TROPICOS (Missouri Botanical Garden). Para la determinación de los especimenes, que se conservan en el herbario USP, hemos consultado los herbarios F, MA, MO.

Los nombres de los sintáxones siguen el Código Internacional de Nomenclatura Fitosociológica (Weber et al. 2000).

\section{RESULTADOS Y DISCUSIÓN}

\section{Novedades fitosociológicas}

A continuación se incluyen la descripción y tipificación de los nuevos sintáxones encontrados en los territorios como resultado de los inventarios levantados. Para cada uno de ellos se comenta su combinación florística, distribución geográfica y su relación con los descritos en otras áreas del Neotrópico.

\section{Hydocleydo nymphoidis-Nymphoidetum herzogii Galán de Mera \& Linares Perea ass. nova}

[Tabla 2, holotypus ass. inv. 2]

Asociación de ninfeidos de aguas someras (10-30 cm de profundidad) más o menos eutrofizadas, presidida por el endemismo boliviano Nymphoides herzogii
A. Galán \& G. Navarro. Como ya comentamos en otra ocasión (Galán de Mera et al. 2006) esta vegetación alterna en el tiempo con las comunidades de Marsilea crotophora D.M. Johnson y la presencia de Utricularia foliosa, U. gibba y U. poconensis Fromm. Utricularia refleja la existencia de ácidos orgánicos (Colonnello, 1995); el aporte de lodos durante la época de lluvias, permite la proliferación de $M$. crotophora, para posteriormente dar paso a los ninfeidos que tienen su óptimo entre junio y agosto, reflejando así una sinfenosucesión en un mismo espacio geomorfológico en el sentido de Pérez Latorre et al. (2004).

El género Nymphoides en América presenta dos centros de diversidad, en el Caribe $-N$. aurea (Britton) Britton, $N$. grayana Kuntze- y en la zona Chaco-Cerrado -N. verrucosa (R.E. Fr.) A. Galán \& G. Navarro, N. herzogii (Galán de Mera \& Navarro, 1989; Borhidi, 1991). N. humboldtiana (Kunth) Kuntze tiene una distribución mucho más amplia extendiéndose por las aguas someras del Neotrópico; asimismo es la única especie que encontramos en la Cuenca Amazónica (Brako \& Zarucchi, 1993). N. herzogii fue descrita con material de los alrededores de Santa Cruz (Bolivia), ahora damos a conocer su localización en los Llanos de Moxos y el Pantanal.

Hydrocleydo-Nymphoidetum herzogii, citada por Haase (1989) en los Llanos de Moxos como comunidad de $N$. humboldtiana y Luziola peruviana Juss. ex J.F. Gmel., es vicariante del Hydrocleydo-Nymphoidetum humboldtianae Galán de Mera \& Navarro 1992 del Chaco y de LudwigioEichhornietum diversifoliae Castroviejo \& López 1985 de los Llanos del Orinoco, con quien comparte especies como Hydrocleys parviflora Seub., Ludwigia sedoides (Bonpl.) H. Hara o Sagittaria guyanensis Kunth (Galán de Mera, 2007), y por su composición 
Tabla 3

\begin{tabular}{|c|c|c|c|c|c|c|c|c|}
\hline \multicolumn{9}{|c|}{$\begin{array}{c}\text { Oxycaryo cubensis-Eleocharitetum acutangulae } \\
\text { Galán de Mera \& Linares Perea ass. nova } \\
\text { (Cladietea jamaicensis, Oryzo-Hymenachnetalia, Hymenachnion amplexicaulis) }\end{array}$} \\
\hline Inventario $\mathrm{n}^{\mathrm{o}}$ & 1 & 2 & 3 & 4 & 5 & 6 & 7 & 8 \\
\hline Área m² & 60 & 50 & 50 & 100 & 100 & 100 & 100 & 70 \\
\hline Profundidad (cm) & 50 & 60 & 70 & 90 & 80 & 80 & 50 & 80 \\
\hline \multicolumn{9}{|l|}{ Características de asociación } \\
\hline Eleocharis acutangula & 1 & 1 & 2 & 4 & 5 & 5 & 5 & 3 \\
\hline Oxycaryum cubense & 4 & 5 & 4 & 3 & 2 & 2 & . & 4 \\
\hline \multicolumn{9}{|c|}{ Características de unidades superiores } \\
\hline Hymenachne amplexicaulis & 1 & 2 & . & 1 & 1 & 1 & 1 & 2 \\
\hline Xyris caroliniana & . & . & . & 1 & 1 & 1 & 1 & 1 \\
\hline Osmunda cinnamomea & . & . & . & 1 & 2 & . & 2 & 1 \\
\hline Cyperus surinamensis & 2 & . & . & . & . & . & . & + \\
\hline Habenaria repens & . & . & . & 1 & . & . & . & . \\
\hline Aeschynomene evenia & . & . & . & + & . & . & . & . \\
\hline Ludwigia hyssopifolia & . & . & . & + & . & . & . & . \\
\hline Echinodorus paniculatus & . & . & . & . & . & + & . & . \\
\hline \multicolumn{9}{|l|}{ Compañeras } \\
\hline Utricularia gibba & 2 & + & + & 1 & . & 1 & . & 1 \\
\hline Pontederia subovata & 2 & 1 & + & . & . & . & . & . \\
\hline Ludwigia helminthorrhiza & 2 & + & . & . & . & . & . & 1 \\
\hline Salvinia auriculata & . & . & . & + & 1 & . & . & 1 \\
\hline Eichhornia azurea & 2 & . & 1 & . & . & . & . & . \\
\hline Marsilea crotophora & 2 & . & 1 & . & . & . & . & . \\
\hline Eichhornia crassipes & . & . & . & 2 & . & . & . & + \\
\hline Pontederia rotundifolia & 1 & . & . & . & . & . & . & . \\
\hline Mimosa pudica & . & . & . & . & . & . & 1 & . \\
\hline
\end{tabular}

Localidades: 1 y 2- Bolivia, Beni, San Borja, Estancia Los Reyes (19L7399); 3- Bolivia, Beni, San Borja, Estancia Los Reyes (19L7294); 4- Venezuela, Apure, Hato E1 Frío, Laguna La Carmera (19N0853); 5 y 6Venezuela, Apure, Hato El Frío, junto a La Clemencia (19N0796); 7 y 8- Venezuela, Apure, Hato El Frío, Matagorda (19N9642).

florística debe ser incluida en la alianza Nelumbo-Nymphaeion amplae Samek \& Moncada 1971.

\section{Oxycaryo cubensis-Eleocharitetum} acutangulae Galán de Mera \& Linares Perea ass. nova

[Tabla 3, holotypus ass. inv. 4]

La colmatación de las lagunas y esteros neotropicales culmina con la aparición de grandes masas flotantes de Ciperáceas que ya habíamos nombrado como comunidad de Xyris caroliniana Walter y Eleocharis acutangula (Roxb.) Schult. (Galán de Mera et al. 2006). Se trata de una asociación de areal muy extenso en América del Sur (Amazonía, Llanos de Moxos, Llanos del Orinoco, Pantanal) donde domina Oxycaryum cubense (Poepp. \& Kunth) Palla acompañado de otras plantas como Habenaria repens Nutt., Osmunda 
Tabla 4

\begin{tabular}{|c|c|c|c|c|c|c|c|}
\hline (Xyridetea & $\begin{array}{l}\text { po my } \\
\text { Galán } \\
\text { nensis, }\end{array}$ & $\begin{array}{l}\text { hylloi } \\
\text { lera \& } \\
\text { chari }\end{array}$ & $\begin{array}{l}\text { eocha } \\
\text { es Per } \\
\text { minin }\end{array}$ & $\begin{array}{l}\text { m mi } \\
\text { s. nov } \\
\text { chino }\end{array}$ & n boli & & \\
\hline Inventario $n^{\circ}$ & 1 & 2 & 3 & 4 & 5 & 6 & 7 \\
\hline Área $\mathrm{m}^{2}$ & 100 & 100 & 100 & 100 & 100 & 50 & 50 \\
\hline Características de asoci & & & & & & & \\
\hline Bacopa myriophylloides & 2 & 1 & 2 & 1 & 2 & 2 & 1 \\
\hline Características de unida & uperio & & & & & & \\
\hline Limnobium laevigatum & 2 & 3 & 3 & 3 & 3 & 2 & 3 \\
\hline Hydrolea spinosa & 1 & 1 & 1 & 2 & 4 & 3 & 2 \\
\hline Diodia kuntzei & 4 & 4 & 4 & . & . & 2 & 1 \\
\hline Eleocharis minima & 1 & . & 1 & 3 & . & 4 & 4 \\
\hline Echinodorus bolivianus & 1 & . & . & + & 1 & 1 & + \\
\hline Compañeras & & & & & & & \\
\hline Aeschynomene scabra & 1 & 1 & 1 & 1 & . & . & . \\
\hline Pontederia parviflora & 1 & . & 1 & 1 & + & . & . \\
\hline Eichhornia azurea & + & . & . & . & . & + & . \\
\hline Hyptis lorentziana & . & + & + & . & . & . & . \\
\hline Pontederia cordata var. & & & & & & & \\
\hline lancifolia & . & . & 1 & . & . & . & . \\
\hline Sagittaria guyanensis & . & . & + & + & . & . & . \\
\hline Utricularia gibba & . & . & . & . & 1 & 1 & . \\
\hline Ludwigia sedoides & . & . & . & . & . & + & + \\
\hline Salvinia auriculata & . & . & . & . & . & + & . \\
\hline Sagittaria rhombifolia & . & . & . & . & . & + & . \\
\hline
\end{tabular}

Localidades: 1 y 2- Bolivia, Santa Cruz, San Matías, Reserva Biológica El Pantanal (21K4057); 3, 4 y 5Bolivia, Santa Cruz, San Matías, Reserva Biológica El Pantanal (21K3856); 6 y 7- Bolivia, Santa Cruz, San Matías, Reserva Biológica Cambará, junto al Gran Curichi fronterizo con Brasil (21K4356).

cinnamomea L. y Xyris caroliniana.

Desde el punto de vista sucesional la colmatación de estos medios se inicia con las asociaciones de Hymenachne amplexicaulis, y a medida que prolifera el entramado herbáceo van siendo sustituidas por Oxycaryum cubense y otras especies (Rial, 2000, 2006).

Bacopo myriophylloidis-Eleocharitetum minimae Galán de Mera \& Linares Perea ass. nova

[Tabla 4, holotypus ass. inv. 6]
Pastizal, caracterizado por plantas anuales, que permanece encharcado en la época seca en los Llanos de Moxos y el Pantanal (fig. 4), siendo la combinación característica de plantas de la asociación Bacopa myriophylloides Wettst., Diodia kuntzei K. Schum., Echinodorus bolivianus (Rusby) Holm-Niels., Eleocharis minima Kunth., Hydrolea spinosa L. y Limnobium laevigatum (ver Beck, 1984).

Se trata de una asociación vicariante del Spilantho uliginosi-Paspaletum orbiculati Castroviejo \& López 1985 de los Llanos del 
Tabla 5

\begin{tabular}{|c|c|c|c|c|c|c|c|c|}
\hline (Cladietea jamai & $\begin{array}{l}\text { noeo } \\
\text { n de } \\
\text { isis, }\end{array}$ & $\begin{array}{l}\operatorname{losa} \\
\text { a \& I } \\
o-H y\end{array}$ & $\begin{array}{l}\text { ennet } \\
\text { rres } \mathrm{P} \\
\text { nachn }\end{array}$ & $\begin{array}{l}\text { acule } \\
\text { ass. } \\
\text { ia, Ip }\end{array}$ & $\begin{array}{l}\boldsymbol{e} \\
\boldsymbol{a} \\
\text { oeion }\end{array}$ & tulosae) & & \\
\hline Inventario $\mathrm{n}^{\circ}$ & 1 & 2 & 3 & 4 & 5 & 6 & 7 & 8 \\
\hline Área $\mathrm{m}^{2}$ & 14 & 50 & 100 & 100 & 100 & 20 & 100 & 100 \\
\hline Profundidad $(\mathrm{cm})$ & . & . & 15 & . & . & . & . & . \\
\hline Características de asociación & & & & & & & & \\
\hline Senna aculeata & 2 & 3 & 4 & 4 & 2 & 4 & 5 & 4 \\
\hline Melochia nodiflora & . & . & . & . & + & + & + & 1 \\
\hline Características de unidades sup & iores & & & & & & & \\
\hline Ipomoea carnea subsp. fistulosa & 1 & 1 & 1 & . & 1 & 1 & + & \\
\hline Thalia geniculata & . & . & . & . & 1 & 1 & 2 & 1 \\
\hline Eleocharis mutata & . & . & . & . & 1 & + & 1 & . \\
\hline Aeschynomene scabra & . & + & 1 & . & . & . & . & . \\
\hline Cyperus flavescens & . & . & . & 1 & . & + & . & . \\
\hline Ludwigia hyssopifolia & . & . & . & 1 & . & . & + & . \\
\hline Ipomoea asarifolia & . & . & . & 1 & . & . & . & 1 \\
\hline Cyperus surinamensis & + & . & . & . & . & . & . & . \\
\hline Hymenachne donacifolia & . & 1 & . & . & . & . & . & . \\
\hline Sagittaria sp. & . & 1 & . & . & . & . & . & . \\
\hline Gymnocoronis spilanthoides & . & . & 1 & . & . & . & . & . \\
\hline Rhynchospora tricuspidata & . & . & 1 & . & . & . & . & . \\
\hline Canna sp. & . & . & . & . & . & . & 2 & . \\
\hline Compañeras & & & & & & & & \\
\hline Mimosa dormiens & . & . & . & 1 & 1 & 1 & + & + \\
\hline Heliotropium indicum & . & . & . & 1 & 1 & 1 & 1 & . \\
\hline Ambrosia peruviana & . & . & . & 3 & 3 & 2 & 1 & . \\
\hline Sida glomerata & . & . & . & . & + & + & + & 1 \\
\hline Senna alata & . & . & . & . & 4 & 3 & 1 & . \\
\hline Solanum hirtum & + & . & . & 1 & . & . & . & . \\
\hline Mikania micrantha & . & 2 & 1 & . & . & . & . & \\
\hline Malachra radiata & . & . & . & 1 & . & 1 & . & \\
\hline Mimosa pudica & . & . & . & . & 1 & . & . & 2 \\
\hline
\end{tabular}

Otras plantas: Indigofera suffruticosa 3, Cynodon dactylon 4 en 1; Melochia arenosa 1, Rhabdadenia pohlii + en 2; Polygonum hydropiperoides 3, Aniseia martinicensis + en 3; Sida sp. + en 4; Diodia teres + en 6; Cochlospermum vitifolium 1 en 7; Helicteres guazumifolia 2, Borreria aristeguietana + , Bauhinia benthamiana + , Sida procumbens + en 8 .

Localidades: 1- Bolivia, Beni, San Borja, Estancia Los Reyes (19L7399); 2 y 3- Bolivia, Beni, San Borja, Estancia Los Reyes (19L7294); 4 y 5- Venezuela, Apure, Hato El Frío, Matagorda (19N9770); 6 y 7 Venezuela, Apure, Hato El Frío, Matagorda (19N9673); 8- Venezuela, Apure, Hato El Frío, La Clemencia (19N0770). 
Tabla 6

\begin{tabular}{|c|c|c|c|c|c|c|}
\hline \multicolumn{7}{|c|}{$\begin{array}{c}\text { Comunidad de Pontederia parviflora } \\
\text { (Cladietea jamaicensis, Scirpo-Eleocharitetalia, Eleocharition) }\end{array}$} \\
\hline Inventario $\mathrm{n}^{\circ}$ & 1 & 2 & 3 & 4 & 5 & 6 \\
\hline Área $\mathrm{m}^{2}$ & 30 & 100 & 100 & 100 & 200 & 200 \\
\hline Profundidad $(\mathrm{cm})$ & 5 & 15 & 30 & 10 & 30 & 30 \\
\hline \multicolumn{7}{|c|}{ Características de la comunidad } \\
\hline Pontederia parviflora & 2 & 5 & 5 & 3 & 3 & 4 \\
\hline \multicolumn{7}{|c|}{ Características de unidades superiores } \\
\hline Rhynchospora tricuspidata & 1 & . & 1 & . & . & . \\
\hline Aeschynomene scabra & . & 1 & 1 & 1 & . & . \\
\hline Eleocharis interstincta & . & 3 & 2 & . & . & . \\
\hline Thalia geniculata & . & . & 1 & 1 & . & . \\
\hline Cyperus giganteus & . & . & . & 1 & 1 & + \\
\hline Ludwigia hyssopifolia & . & . & . & . & + & . \\
\hline Ludwigia tomentosa & . & . & . & . & + & . \\
\hline Sagittaria rhombifolia & . & . & . & . & 1 & + \\
\hline \multicolumn{7}{|l|}{ Compañeras } \\
\hline Marsilea crotophora & 3 & . & . & . & . & . \\
\hline Pontederia subovata & . & 1 & 1 & . & . & + \\
\hline Salvinia auriculata & . & 1 & 1 & . & + & 2 \\
\hline Eichhornia azurea & . & . & . & 1 & 1 & + \\
\hline
\end{tabular}

Localidades: 1-3- Bolivia, Beni, San Borja, Estancia Los Reyes (19L7398); 4- Bolivia, Santa Cruz, San Matías, Reserva Biológica El Pantanal, humedal al O de la casa (21K4057); 5 y 6- Bolivia, Santa Cruz, San Matías, Reserva Biológica El Pantanal, junto al Gran Curichi fronterizo con Brasil (21K4356).

Orinoco y del Lindernio crustaceaeXyridetum savanensis Galán de Mera 1995 de la Cuenca Amazónica.

Como ya sugeríamos en otro trabajo (Galán de Mera et al. 2006) la alianza Oryzion perennis Galán de Mera et al. 2006 reúne a este tipo de pastizales en Centroamérica y N de América del Sur, lo que hace necesario crear la nueva alianza Echinodorion boliviani Galán de Mera \& Linares Perea all. nova (características: Bacopa myriophylloides, Echinodorus bolivianus; holotypus: Bacopo myriophylloidis-Eleocharitetum minimae Galán de Mera \& Linares Perea ass. nova), que abarca a las comunidades situadas más al sur de la Cuenca Amazónica. Oryzion perennis y Echinodorion boliviani son las dos alianzas que, por el momento, constituyen el orden Eleocharitetalia minimae Galán de Mera et al. 2006.

\section{Ipomoeo fistulosae-Sennetum aculeatae}

Galán de Mera \& Linares Perea ass. nova

[Tabla 5, holotypus ass. inv. 4]

Una de las formas más comunes que tienen los llaneros de las sabanas americanas para favorecer los pastos son las quemas controladas (Sarmiento \& Monasterio, 1969; Beck, 1984; Navarro \& Maldonado, 2005). Senna aculeata (Pohl ex Benth.) H.S. Irwin \& Barneby, arbusto de aproximadamente 1 $\mathrm{m}$, forma parte de la vegetación nitrófila ligada al fuego sobre suelos inundables 
durante la época de lluvias, por lo que en muchas ocasiones aparece acompañada de Ipomoea carnea subsp. fistulosa (Mart. ex Choisy) D.F. Austin; por tanto, incluimos a esta asociación neotropical en Ipomoeion fistulosae (Fuentes \& Navarro, 2000).

Aunque no hemos encontrado este tipo de comunidades en la Cuenca Amazónica, su variabilidad es muy pequeña por lo que consideramos que se trata de la misma asociación en todos los territorios estudiados.

\section{Comunidad de Pontederia parviflora}

[Tabla 6]

Las comunidades acuáticas y palustres con Pontederiáceas son muy frecuentes en el Neotrópico (Galán de Mera \& Vicente
Orellana, 2006). Sin embargo, mientras que las comunidades con Pontederia subovata son muy escasas en los Llanos del Orinoco, en los llanos bolivianos podemos observar áreas dominadas por $P$. parviflora Alexander y $P$. cordata var. lancifolia (Muhl.) Torr. en aguas someras $(15-30 \mathrm{~cm})$ sobre suelos limoarenosos en contacto con los yomomales de Cyperus giganteus Vahl (fig. 4).

P. parviflora y P. cordata var. lancifolia curiosamente tienen una distribución mesoamericana pero vuelven a reaparecer en las cuencas de los ríos Beni, Paraguay y Paraná (Lowden, 1973; Pott \& Pott, 2000). Con la misma fisionomía hemos observado comunidades con $P$. cordata var. cordata (Echinodoro grandiflori-Pontederietum cordatae) al S de Paraguay (Galán de Mera

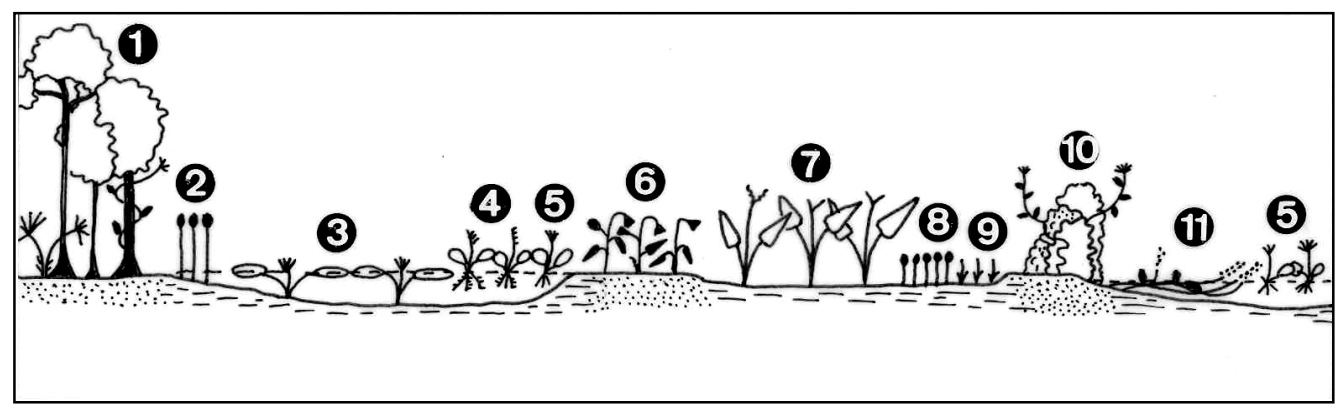

Figura 3. Esquema de la vegetación en los humedales de la Estación Biológica El Frío. 1- Bosques semidecíduos de los Llanos Occidentales del Orinoco (Bromelio-chrysanthae-Platymiscietum pinnati), 2- juncal de aguas profundas (Eleocharitetum interstinctae), 3-Comunidades de ninfeidos (Ludwigio sedoidis-Eichhornietum diversifoliae, 4- "Borales" de aguas dulces oligótrofas o mesótrofas (Eichhornietum azureae), 5- "Borales" de aguas éutrofas (Eichhornietum crassipedis), 6- Vegetación hidronitrófila de reposaderos (Mimoso chaetosphero-Ipomoeetum fistulosae), 7- Grandes helófitos sobre suelos arcillosos (Thalietum geniculatae), 8- Juncal de aguas poco profundas (Eleocharitetum mutatae), 9- Pastizales inundables (Spilantho uliginosi-Paspaletum orbiculati), 10- Arbusteda pionera de Randio venezuelensis-Annonetum jahnii (variante húmeda con lianas), 11- Graminales flotantes (Luziolo-Hymenachnetum amplexicaulis). Vegetation scheme in the wetlands in El Frio Biological Station. 1-Semideciduous forests of the Occidental Llanos of the Orinoco (Bromelio-chrysanthaePlatymiscietum pinnati), 2- Rushy of deep water (Eleocharitetum interstinctae), 3- water lily communities (Ludwigio sedoidis-Eichhornietum diversifoliae, 4- "Borales" of oligotrophic and mesotrophic fresh water (Eichhornietum azureae), 5- "Borales" of eutrophic fresh water (Eichhornietum crassipedis), 6- hydronitrophilous vegetation of sleeping places (Mimoso chaetosphero-Ipomoeetum fistulosae), 7- High helophytic vegetation on clayey soils (Thalietum geniculatae), 8-Rushy of shallow water (Eleocharitetum mutatae), 9- Flooding pastures (Spilantho uliginosi-Paspaletum orbiculati), 10-Pioneer shrubs of Randio venezuelensis-Annonetum jahnii (humid variant with lianas), 11- Floating grasslands (Luziolo-Hymenachnetum amplexicaulis). 
\& Navarro, 1992); sin embargo, no conocemos su presencia en la región del Caribe (Borhidi et al. 1983).

\section{Sinfitocorología}

En los territorios estudiados la vegetación climatófila está constituida por bosques semidecíduos de fisionomía muy parecida, y por una serie de comunidades, en muchos casos comunes, geovicarias hacia los suelos más inundables (fig. 3 y 4).

En la tabla 7 damos a conocer las comunidades vegetales observadas en las tres áreas estudiadas.

Los bosques con inundación de los Llanos del Orinoco contienen Copernicia tectorum (Kunth) Mart., mientras que en los llanos de Bolivia esta palmera es sustituida por asociaciones de C. alba con Tabebuia heptaphylla. Las arbustedas del Orinoco temporalmente inundables (NectandroViticion orinocensis Galán de Mera et al.
2006) incluyen especies comunes con las bolivianas, como Arrabidaea corallina (Jacq.) Sandwith, Bauhinia glabra Jacq., Cissampelos pareira L., Guazuma ulmifolia Lam., Paullinia pinnata L. o Sapium glandulosum (L.) Morong, y especies geovicarias que se distribuyen a un lado y a otro de la Cuenca Amazónica (tab. 8).

Las comunidades ligadas a los medios acuáticos y palustres se hacen comunes en ambas sabanas aunque nos encontramos con el carácter endémico del HydrocleydoNymphoidetum herzogii en Bolivia, los bajíos con Bacopo myriophylloidisEleocharitetum minimae -sobre todo en el patanal boliviano-brasileño- y los yomomales de Rhabdadenio-Cyperetum gigantei de los humedales del Chaco y el Cerrado frente al caribeño-mesoamericano Thalietum geniculatae. En los llanos bolivianos hay que resaltar a las comunidades de Pontederia parviflora que

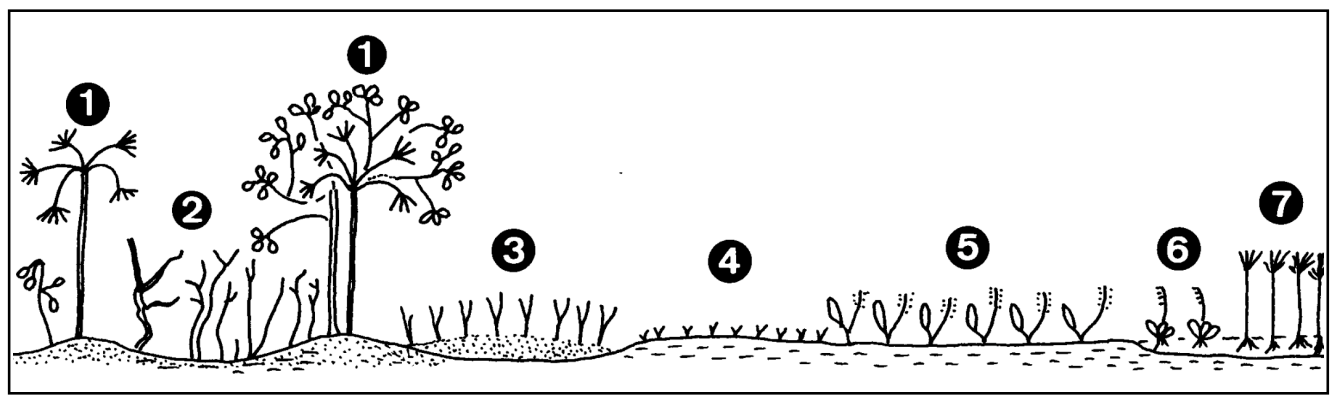

Figura 4. Esquema de la vegetación en los humedales de la Reserva Biológica El Pantanal. 1- Palmar de áreas elevadas con Copernicia alba y Tabebuia heptaphylla, 2-Chaparral de Curatella americana y Licania parvifolia, 3- Pastizal de áreas elevadas con Paspalum plicatulum, P. wrightii y Andropogon bicornis en las zonas degradadas, 4- Pastizal de áreas inundables (Bacopo-Eleocharitetum minimae), 5- Comunidades de Pontederia parviflora de aguas someras, 6- "Taropales" de Eichhornietum azureae de aguas oligótrofas o mesótrofas, 7 "Yomomales" con inundación permanente (RhabdadenioCyperetum gigantei). Vegetation scheme in the wetlands of El Pantanal Biological Reserve. 1- Palm grove on elevated areas of Copernicia alba and Tabebuia heptaphylla, 2-Chaparral of Curatella americana and Licania parvifolia, 3- Pasture on elevated areas with Paspalum plicatulum, P. wrightii (in disturbed zones with Andropogon bicornis), 4-Flooding pastures (Bacopo-Eleocharitetum minimae), 5- Pontederia parviflora communities of shallow water, 6- "Taropales" of oligotrophic and mesotrophic fresh water (Eichhornietum azureae), "Yomomales" with permanent inundation (Rhabdadenio-Cyperetum gigantei). 


\begin{tabular}{|l|l|l|}
\hline \multicolumn{1}{|c|}{ Comunidades } & $\begin{array}{c}\text { Venezuela } \\
\text { (Llanos del } \\
\text { Orinoco) }\end{array}$ & $\begin{array}{c}\text { Bolivia } \\
\text { (Moxos, } \\
\text { Pantanal) }\end{array}$ \\
\hline Bacopo myriophylloidis-Eleocharitetum minimae & & \\
\hline Coccolobietea obtusifoliae & & \\
\hline Comunidad de Curatella americana y Licania parviflora & & \\
\hline Comunidad de Bromelia serra con Copernicia tectorum & & \\
\hline Comunidad de Lemna aequinoctiali s & & \\
\hline Comunidad de Nectandra pichurim y Vitexorinocensi s & & \\
\hline Comunidad de Pontederia parviflora & & \\
\hline Comunidad de Tabebuia insigni s & & \\
\hline Eichhornietum azureae & & \\
\hline Eichhornietum crassipedis & & \\
\hline Oxycaryo cubensis-Eleocharitetum acutangulae & & \\
\hline Eleocharitetum interstinctae & & \\
\hline Hydrocleydo-Nymphoidetum herzogii & & \\
\hline Ipomoeo fistulosae-Sennetum aculeatae & & \\
\hline Ludwigio sedoidis-Eichhornietum diversifoliae & & \\
\hline Luziolo spruceanae-Hymenachnetum amplexicaulis & & \\
\hline Mimoso chaetosphero-Ipomoeetum fistulosae & & \\
\hline Palmerales de Tabebuia heptaphylla y Copernicia alba & & \\
\hline Rhabdadenio-Cyperetum gigantei & & \\
\hline Spilantho uliginosi-Paspaletum orbicu lati & & \\
\hline Thalietum geniculatae & & \\
\hline
\end{tabular}

Tabla 7. Comunidades vegetales acuáticas y palustres de los Llanos del Orinoco (Venezuela), Moxos y Pantanal (Bolivia). Aquatic and trowel vegetal communities of the Llanos of the Orinoco (Venezuela), Moxos and Pantanal (Bolivia).

pueblan planicies limo-arenosas y están ausentes de los Llanos del Orinoco.

Por fin, las arbustedas de Coccoloba obtusifolia Jacq., que soportan caudales con grandes oscilaciones en los Llanos del Orinoco, son sustituidas por las de Tabebuia insignis (Miq.) Sandwith en los llanos bolivianos.

\section{CONCLUSIONES}

Como conclusión, damos a conocer el esquema sintaxonómico comentado de las comunidades vegetales observadas en Bolivia, las de los Llanos del Orinoco están descritas en Galán de Mera et al. (2006).
I. Vegetación de pleustohelófitos, pleustófitos e hidrófitos

PISTIO STRATIOTIDIS-EICHHORNIETEA CRASSIPEDIS O. Bolòs, Cervi \& Hatschbach 1991

+ Pistio stratiotidis-Eichhornietalia crassipedis O. Bolòs, Cervi \& Hatschbach 1991

* Pistio stratiotidis-Eichhornion crassipedis O. Bolòs, Cervi \& Hatschbach 1991

1. Eichhornietum crassipedis Samek \& Moncada 1971 [Pleustohelófitos de aguas éutrofas]

* Eichhornion azureae Borhidi \& Muñiz ex Galán de Mera \& Navarro 1992

2. Eichhornietum azureae Borhidi in Borhidi, Muñiz \& Del Risco 1983 


\begin{tabular}{cc}
\hline Llanos del Orinoco & Llanos de Bolivia (Moxos, Pantanal) \\
\hline Cissus elata & Cissus spinosa \\
Combretum laxum & Combretum lanceolatum \\
Cordia sagotii & Cordia naidophila \\
Davilla kunthii & Davilla elliptica \\
Desmoncus orthacanthos & Desmoncus cuyabaensis \\
Eugenia monticola & Eugenia florida \\
Licania octandra & Licania parviflora \\
\hline
\end{tabular}

Tabla 8. Especies vicariantes entre los Llanos del Orinoco y los llanos bolivianos. Vicariant species between the Llanos of the Orinoco and the Bolivian plains.

[Pleustohelófitos de aguas dulces oligótrofas a mesótrofas]

LEMNETEA MINORIS R.Tx. ex O. Bolòs \& Masclans 1955

+ Lemnetalia aequinoctialis Schwabe-Braun \& R.Tx. 1981 ex Galán de Mera \& Navarro 1992

* Azollo carolinianae-Salvinion auriculatae Borhidi \& Muñiz in Borhidi, Muñiz \& Del Risco (1979) 1983

3. Comunidad de Lemna aequinoctialis [Lentejas de aguas éutrofas]

CABOMBO-NYMPHAEETEA Borhidi \& Del Risco in Borhidi, Muñiz \& Del Risco (1979) 1983

+ Nymphaeetalia amplae Knapp ex Borhidi 1996

* Nelumbo-Nymphaeion amplae Samek \& Moncada 1971

4. Hydrocleydo nymphoidis-Nymphoidetum herzogii Galán de Mera \& Linares Perea ass. nova [Comunidades de ninfeidos de los llanos bolivianos]

II. Comunidades helofíticas y pastizales

CLADIETEA JAMAICENSIS Knapp ex Borhidi 1996

+ Scirpo-Eleocharitetalia interstinctae Borhidi \& Muñiz in Borhidi 1996

* Eleocharition interstincto-mutatae
Castroviejo \& López 1985

5. Eleocharitetum interstinctae Castroviejo \& López 1985 [Juncal de aguas profundas] 6. Comunidades de Pontederia parviflora [Comunidades helofíticas de aguas someras] * Typhion domingensis Del Risco in Borhidi 1996

7. Rhabdadenio ragonesei-Cyperetum gigantei Galán de Mera \& Navarro 1992 [Asociación de grandes helófitos]

+ Oryzo grandiglumis-Hymenachnetalia amplexicaulis Galán de Mera \& Rosa in Galán de Mera, Rosa \& Cáceres 2002

* Hymenachnion amplexicaulis Galán de Mera 1995

8. Luziolo spruceanae-Hymenachnetum amplexicaulis Susach Campalans 1989 [Graminales flotantes]

9. Oxycaryo cubensis-Eleocharitetum acutangulae Galán de Mera \& Linares Perea ass. nova [Grandes masas flotantes de Ciperáceas]

* Ipomoeion fistulosae Fuentes \& Navarro 2000, prov.

10. Mimoso chaetosphero-Ipomoeetum fistulosae Fuentes \& Navarro 2000 [Vegetación hidronitrófila de reposaderos] 11. Ipomoeo fistulosae-Sennetum aculeatae Galán de Mera \& Linares Perea ass. nova [Vegetación nitrófila ligada al fuego]

XYRIDETEA SAVANENSIS Galán de Mera 1995 
+ Eleocharitetalia minimae Galán de Mera, González, Morales, Oltra \& Vicente Orellana 2006

* Echinodorion boliviani Galán de Mera \& Linares Perea all. nova

12. Bacopo myriophylloidis-Eleocharitetum

minimae Galán de Mera \& Linares Perea ass. nova [Pastizal inundable rico en plantas anuales]

AGRADECIMIENTOS. Deseamos expresar nuestro agradecimiento a las personas e instituciones que, con su apoyo y ayuda logística, nos han facilitado la realización de este trabajo: Javier Castroviejo (Fundación Amigos del Coto de Doñana, Sevilla), José Ayarzagüena y Antonio Castro (Fundación Hombre y Naturaleza, Santa Cruz, Bolivia), Fundación Amigos del Coto de Doñana- Fundación Carolina-Universidad San Pablo-CEU (Máster en Gestión y Conservación de la Biodiversidad en los Trópicos, Madrid), familias Calle y Mundaca (San Borja, Bolivia), Guzmán (San Matías, Bolivia) y Maldonado (Hato El Frío, Venezuela).

\section{BIBLIOGRAFÍA}

ADÁMOLI, J. -1999- Los humedales del Chaco $y$ del Pantanal. Universidad de Buenos Aires.

BECK, S.G. -1984- Comunidades vegetales de las sabanas inundadizas del NE de Bolivia. Phytocoenologia 12(2/3): 321-350.

BOELCKE, O., D.M. MOORE y F.A. ROIG 1985- Transecta Botánica de la Patagonia Austral. Consejo Nacional de Investigación Científica y Técnica de Argentina-Instituto de la Patagonia de Chile-Royal Society, Buenos Aires-Londres.

BORHIDI, A. -1991- Phytogeography and Vegetation Ecology of Cuba. Akadémici Kiadó, Budapest.

BORHIDI, A., O. MUÑIZ \& E. DEL RISCO 1983- Plant communities of Cuba, I. Fresh and salt water, swamp and coastal vegetation. Acta Bot. Hung. 29: 337-376.

BRAKO, L. y J.L. ZARUCCHI -1993- Catálogo de las Angiospermas y Gimnospermas del Perú. Missouri Botanical Garden, St. Louis.
BRAUN-BLANQUET, J. -1964- Pflanzensoziologie, Grundzüge der Vegetationskunde. Springer Verlag, Berlin, Wien, New York.

CASTROVIEJO, S. y G. LÓPEZ -1985- Estudio y descripción de las comunidades vegetales del "Hato El Frío". Los Llanos de Venezuela. Mem. Soc. Ci. Nat. La Salle 45: 79-151.

CLEEF, A.M. -1981- The Vegetation of the Páramos of the Colombian Cordillera Oriental. J. Cramer, Vaduz.

COLONNELLO, G. -1995- La vegetación acuática del delta del río Orinoco (Venezuela). Composición florística y aspectos ecológicos (I). Mem. Soc. Ci. Nat. La Salle 55: 3-34.

CONVENTION ON WETLANDS -2007www.ramsar.org.

DALY, D.C. \& J.D. MITCHELL -2000- Lowland vegetation of tropical South America- an overview: 391-454. In D. Lentz (ed.): Imperfect Balance: Landscape Transformations in the pre-Columbian Americas. Columbia University Press, New York.

DEIL, U. -2005-A review on habitats, plant traits and vegetation of ephemeral wetlands- a global perspective. Phytocoenologia 35(2-3): 533-705.

DIERSCHKE, H. -1994- Pflanzensoziologie. Ulmer, Stuttgart.

ENCARNACIÓN, F. -1985- Introducción a la flora y vegetación de la Amazonía peruana: estado actual de los estudios, medio natural y ensayo de una clave de determinación de las formaciones vegetales en la llanura amazónica. Candollea 40: 237-252.

FRANCO, P., O. RANGEL y G. LOZANO -1986Estudios ecológicos en la Cordillera OrientalII. Las comunidades vegetales de los alrededores de la laguna de Chingaza (Cundinamarca). Caldasia 15: 71-75.

FUENTES, A. y G. NAVARRO -2000- Estudio fitosociológico de la vegetación de una zona de contacto Chaco-Cerrado en Santa Cruz (Bolivia). Lazaroa 21: 73-109.

GALÁN DE MERA, A. -1995- Ensayo sintaxonómico sobre las comunidades vegetales acuáticas del Perú. Arnaldoa 3(1): 51-58.

GALÁN DE MERA, A. -2007-Flora y Vegetación de la Estación Biológica El Frio (Llanos Occidentales del Orinoco, Apure, 
Venezuela). Publicaciones de la Asociación Amigos del Coto de Doñana, Sevilla.

GALÁN DE MERA, A., S. BALDEÓN, H. BELTRÁN, M. BENAVENTE y J. GÓMEZ 2004- Datos sobre la vegetación del centro del Perú. Acta Bot. Malacitana 29: 89-115.

GALÁN DE MERA, A., C. CÁCERES y A. GONZÁLEZ -2003- La vegetación de la alta montaña andina del sur del Perú. Acta Bot. Malacitana 28: 121-147.

GALÁN DE MERA, A., A. GONZÁlEZ, R. MORALES, B. OLTRA y J.A. VICENTE ORELLANA -2006- Datos sobre la vegetación de los Llanos Occidentales del Orinoco (Venezuela). Acta Bot. Malacitana 31: 97-129.

GALÁN DE MERA, A. y G. NAVARRO -1989Nymphoides (Menyanthaceae) en Bolivia y Paraguay. Opusc. Bot. Pharm. Complutensis 5: 79-86.

GALÁN DE MERA, A. y G. NAVARRO -1992Comunidades vegetales acuáticas del Paraguay occidental. Caldasia 17: 35-46.

GALÁN DE MERA, A., M.V. ROSA y C. CÁCERES -2002- Una aproximación sintaxonómica sobre la vegetación del Perú. Clases, órdenes y alianzas. Acta Bot. Malacitana 27: 75-103.

GALÁN DE MERA, A. y J.A. VICENTE ORELLANA -2006- Aproximación al esquema sintaxonómico de la vegetación de la región del Caribe y América del Sur. Anales de Biología 28: 3-27.

GANDULLO, R. y A.M. FAGGI -2005Interpretación sintaxonómica de los humedales del noroeste de la provincia de Neuquén, Argentina. Darwiniana 43(1-4): 1029.

HAASE, R. -1989- Plant communities of a savanna in northern Bolivia I. Seasonally flooded grassland and gallery forest. Phytocoenologia 18: 55-81.

HAASE, R. -1990- Plant communities of a savanna in northern Bolivia II. Palm swamps, dry grasslands and shrublands. Phytocoenologia 18: 343-370.

HAUENSTEIN, E., M. GONZÁLEZ, F. PEÑACORTÉS y A. MUÑOZ-PEDREROS -2002Clasificación y caracterización de la flora y vegetación de los humedales de la costa de
Tolten (IX Región, Chile). Gayana Bot. 59(2): 87-100.

KALLIOLA, R., M. PUHAKKA y W. DANJOY -1993- Amazonía Peruana. Vegetación húmeda tropical en el llano subandino. Universidad de Turku-ONERN, Jyväskylä.

KILLEEN, J., E. EMILIA GARCÍA y S.G. BECK -1993- Guía de árboles de Bolivia. Herbario Nacional de Bolivia-Missouri Botanical Garden, La Paz-St. Louis.

LINARES-PALOMINO, R., R.T. PENNINGTON \& S. BRIDGEWATER -2003- The phytogeography of the seasonally dry tropical forests in Equatorial Pacific South America. Candollea 58: 473-499.

LINARES PEREA E. -2000-Vegetación del Santuario Nacional Lagunas de Mejía, Arequipa-Perú. Universidad Nacional de San Agustín, Arequipa.

LOWDEN, R.M. -1973- Revisión of the genus Pontederia L. Rhodora 75: 426-487.

MÜLLER, G.K. \& P. GUTTE -1985- Beiträge zur Kenntnis der Vegetation der Flussauen, Sümpfe und Gëwasser der zentralperuanischen Küstenregion. Wiss. Z Karl-Marx-Univ. Leipzig, Math--Naturwiss. R. 34: 410-429.

MÜLLER, M.J. -1982- Selected climatic data for a global set of standard stations for vegetation science. Dr. W. Junk Publishers, The Hague, Boston, London.

NAVARRO, G. y M. MALDONADO -2005Geografía ecológica de Bolivia. Vegetación y Ambientes Acuáticos. Fundación Simón I. Patiño, Santa Cruz.

PENNINGTON, R.T., G.P. LEWIS \& J.A. RATTER -2006- Neotropical Savannas and Seasonally Dry Forests. CRC Press, New York.

PÉREZ LATORRE, A.V., D. NAVAS FERNÁNDEZ, Ó. GAVIRA, G. CABALLERO y B. CABEZUDO -2004Vegetación del Parque Natural de las sierras Tejera, Almijara y Alhama (Málaga-Granada, Andalucía, España). Acta Bot. Malacitana 29: 117-190.

POTT, V., N. BUENO, R. PEREIRA, R. SALIS \& N. VIEIRA -1989- Distribuçao de macrófitas aquáticas numa lagoa na Fazenda Nhumirim, Nhecolândia, Pantanal, MS. Acta 
Bot. Bras. 3: 153-168.

POTT, A. \& V.J. POTT -1994- Plantas do Pantanal. Embrapa, Corumbà.

POTT, V.J. \& A. POTT -2000- Plantas Aquáticas do Pantanal. Embrapa, Corumbà.

POUILLY, M., S.G. BECK, M. MORAES y C. IBÁÑEZ -2004- Diversidad biológica en la llanura de inundación del Río Mamoré. Fundación Simón I. Patiño, Santa Cruz.

PRANCE, G.T. \& G.B. SCHALLER -1982Preliminary study of some vegetation types of the Pantanal, Mato Grosso. Brazil. Brittonia 32: 228-251.

RANGEL, O. y J. AGUIRRE -1983-Comunidades acuáticas altoandinas-I. Vegetación sumergida y de ribera en el lago de Tota, Boyacá, Colombia. Caldasia 13(65): 719-742.

RANGEL, O., P.D. LOWY y M. AGUILAR 1997- Tipos de vegetación en Colombia. Colombia Diversidad Biótica II. Universidad Nacional de Colombia, Santafé de Bogotá.

RATTER, J.A., A. POTT, V.J. POTT, C.N. CUNHA \& M. HARIDASAN -1988Observations on woody vegetation types in the Pantanal and at Corumbá, Brazil. Notes Roy. Bot. Gard. Edinburgh 45: 503-525.

RIAL, A. -2000-Aspectos cualitativos de la zonación y estratificación de comunidades de plantas acuáticas en un humedal de los Llanos de Venezuela. Mem. Fund. La Salle Ci. Nat. $60: 69-85$.

RIAL, A. -2006- Variabilidad espacio-temporal de las comunidades de plantas acuáticas en un humedal de los Llanos de Venezuela. Rev. Biol. Trop. 54(2): 403-413.

RIVAS-MARTÍNEZ, S. -2005- Global Bioclimatics (www.globalbioclimatics.org).

RIVAS-MARTÍNEZ, S., D. SÁNCHEZ-MATA \&
M. COSTA -1999- North American Boreal and Western temperate Forest Vegetation (Syntaxonomical sinopsis of the potential natural plant communities of North America, II). Itinera Geobot. 12: 5-316.

SAN MARTÍN PADOVANI, C., R. MEDINA JARAMILLO, P. OJEDA NEMPU y C. RAMÍREZ GARCÍA -1993- La biodiversidad vegetacional del santuario de la naturaleza "Río Cruces" (Valdivia, Chile). Acta Bot. Malacitana 18: 259-279.

SARMIENTO, G. \& M. MONASTERIO -1969Studies on the savanna vegetation of the Venezuelan Llanos. I. The use of the association-analysis. J. Ecol. 57: 169-598.

SEIBERT, P. \& X. MENHOFER -1991- Die Vegetation des Wohngebietes der Kallawaya und des Hochlandes von Ulla-Ulla in den bolivianischen Anden. Phytocoenologia 20(2): 145-276.

SEIBERT, P. \& X. MENHOFER -1992- Die Vegetation des Wohngebietes der Kallawaya und des Hochlandes von Ulla-Ulla in den bolivianischen Anden. Phytocoenologia 20(3): 145-276.

STEYERMARK, J.A., P. BERRY, H. BRUCE \& K. YATSKIEVYCH -1995-2005- Flora of the Venezuelan Guayana. Missouri Botanical Garden, St. Louis.

WEBER, H.E., J. MORAVEC \& J.P. THEURILLAT -2000- International Code of Phytosociological Nomenclature. 3rd edition. J. Veg. Sci. 11: 739-768.

ZEILHOFER, P. \& M. SCHESSL -1999Relationship between vegetation and environmental conditions in the northern Pantanal of Mato Grosso, Brazil. J. Biogeogr. 27: 159-168. 